\title{
Blood Flow Restriction Enhances Rehabilitation and Return to Sport: The Paradox of Proximal Performance
}

\author{
Corbin Hedt, P.T., D.P.T., Patrick C. McCulloch, M.D., Joshua D. Harris, M.D., and \\ Bradley S. Lambert, Ph.D.
}

\begin{abstract}
The use of blood flow restriction (BFR) within rehabilitation is rapidly increasing as further research is performed elucidating purported benefits such as improved muscular strength and size, neuromuscular control, decreased pain, and increased bone mineral density. Interestingly, these benefits are not isolated to structures distal to the occlusive stimulus. Proximal gains are of high interest to rehabilitation professionals, especially those working with patients who are limited due to pain or postsurgical precautions. The review to follow will focus on current evidence and ongoing hypotheses regarding physiologic responses to BFR, current clinical applications, proximal responses to BFR training, potential practical applications for rehabilitation and injury prevention, and directions for future research. Interestingly, benefits have been found in musculature proximal to the occlusive stimulus, which may lend promise to a greater variety of patient populations and conditions. Furthermore, an increasing demand for BFR use in the sports world warrants further research for performance research and recovery. Level of Evidence: Level V, expert opinion.
\end{abstract}

B lood flow restriction (BFR) training has received increasing attention throughout scientific literature and mainstream media as its significance grows in the rehabilitation industry. Reported benefits include improved muscle strength, ${ }^{1-4}$ mitigation of posttraumatic atrophy, 2,5,6 heightened neuromuscular

From Houston Methodist Orthopedics $\theta$ Sports Medicine, Houston, Texas, U.S.A.

The authors report the following potential conflicts of interest or sources of funding: P.C.M. reports board membership, Journal of Knee Surgery, consultancy, Smith $\theta$ Nephew, grants from Siemens, speaker's bureau, Vericel/Aastroom Biosciences, and support from DePuy and Arthrex, outside the submitted work. J.D.H. reports board membership, American Academy of Orthopaedic Surgeons, American Orthopaedic Society for Sports Medicine, $A A N A$, and International Society of Arthroscopy, Knee Surgery, and Orthopaedic Sports Medicine; consultancy for Smith o Nephew; money from SLACK and Xodus Medical; and stock/stock options in PatientPop, outside the submitted work. B.S.L. reports grants from Defli Medical, outside the submitted work. Full ICMJE author disclosure forms are available for this article online, as supplementary material.

Received August 9, 2021; accepted September 15, 2021.

Address correspondence to Corbin Hedt, P.T., D.P.T., S.C.S., C.S.C.S., Houston Methodist Orthopedics $\theta$ Sports Medicine, 5505 West Loop South, Houston, TX 77081.E-mail: Chedt@houstonmethodist.org

(C) 2021 The AUTHORS. Published by Elsevier Inc. on behalf of the Arthroscopy Association of North America. This is an open access article under the CC BY license (http://creativecommons.org/licenses/by/4.0/).

2666-061X/211140

https://doi.org/10.1016/j.asmr.2021.09.024 activity, $^{7}$ decreased pain signaling, ${ }^{8-10}$ and increased bone mineral density. ${ }^{11}$ Importantly, these effects have been most reported to occur in the regions distal to the site of occlusion (commonly applied at the most proximal points of the extremities). However, recent studies have highlighted promise in benefit to the proximal structures as well. ${ }^{12-15}$

When considering the shoulder or hip, one can appreciate the delicate balance in mobility and stability required by the proximal limbs. The glenohumeral and scapulothoracic regions provide a complex synergy of muscle actions in an effort to push, pull, and rotate to an exceedingly high degree, especially with high-level sports such as throwing, gymnastics, and weight-lifting. ${ }^{16}$ The lumbopelvic region ensures a similar means of dynamic stability, but typically in more weightbearing positions and with impact-related activities such as yoga, running, jumping, or dancing. ${ }^{17}$ In the rehabilitation and physical therapy environments, the proximal aspect of a given limb assumes a major role in most rehabilitation programs. While the use of BFR limits occlusion to distal limbs beyond a tourniquet cuff, the potential for proximal benefit seems promising. However, further research is needed to characterize the exact nature of proximal responses of BFR training and to what degree it may be applied in clinical rehabilitation settings or for injury prevention. 
Originally adapted from early studies on muscular occlusion, the initial literature heavily focused on the effectual consequences of vascular manipulation with exercise. ${ }^{18-20}$ The moniker KAATSU training is prominent from research in the late 1990s and early 2000s. These works set the foundation for modern practices and clinical paradigms. While the technology was rudimentary and the occlusive stimuli were rather ambiguous, these studies have allowed for the refinement of current protocols to ensure safety and efficacy with clinical practice.

As rehabilitation professionals adopt and implement BFR into their practice, we must be fastidious in our evaluation of the science. Often with novel technology and techniques, clinical adoption can be antecedent to strong evidence and systematic study. Although BFR is commonly applied in clinical settings, a high degree of speculation remains present in the literature regarding the efficacy of BFR for tissues proximal to the site of occlusion and the potential mechanisms that may play a role. The review to follow will focus on current evidence and ongoing hypotheses regarding physiologic responses to BFR, current clinical applications, proximal responses to BFR training, potential practical applications for rehabilitation and injury prevention, and directions for future research.

\section{Physiologic Mechanisms: An Overview}

In summary, the combination of BFR and lowintensity resistance exercise (BFR-LIX; often defined as exercise performed below $\sim 30 \%$ maximal effort) has often been reported to elicit physiologic responses that are somewhat similar to high-intensity exercise ( $>\sim 70 \%$ maximal effort) with regards to exerciseinduced muscle anabolism and various improvements in muscle performance (e.g., functional capacity, strength). ${ }^{2,21-23}$ The clinical interest in this phenomenon relates to the capacity to provide a sufficient exercise stimulus with reduced mechanical load that may be performed in the early stages following injury or in the early postoperative period after surgery. ${ }^{1,11,24,25}$

Although the need for further research remains, the acute and chronic skeletal muscle responses to BFR have heavily characterized in numerous basic and applied investigations as well as summarized in a number of systematic reviews and meta-analyses with regard to musculature distal to the site of occlusion. $^{2,21,26-33}$ To summarize, the skeletal muscle response to BFR-LIX has been hypothesized to be caused by a combination of intramuscular metabolic stress sensing, metabolite accumulation, mechanotransduction signaling (via muscle contractile activity and cell swelling caused by occlusion), hormonal responses to exercise, intracellular hypoxia, and inflammatory signaling mechanisms. ${ }^{21,31,34,35}$

\section{Effects of BFR Distal to the Occlusion Site}

\section{Skeletal Muscle Responses to Resistance Exercise}

Regarding the responses of skeletal muscle to BFR exercise and training, the majority of investigations have focused on musculature distal to the site of occlusion. To summarize, BFR-LIX has been shown to acutely induce skeletal muscle anabolism through stimulation of mammalian target of rapamycin complex 1 and mitogen-activated protein kinase intracellular signaling pathways, which are both key regulators of skeletal muscle growth and/or remodeling. ${ }^{29-31,36,37}$ Both pathways also are required to elicit maximal protein synthesis rates and can operate in an independent or integrated fashion responding to contractile activity (exercise), cell stress, nutrition, intracellular energy availability, hormone signaling (e.g., insulin and insulin-like growth factors, [IGFs]), and cytokine/ myokine signaling. In the case of injury or the postoperative period, anabolic signaling has been observed to be suppressed due to unloading/inactivity resulting in a loss of muscle mass and function. $28,32,38-41$ Increased protein degradation also has been implicated to play a role in the sarcopenic effects of unloading. ${ }^{42}$ Therefore, because of its anabolic effects with reduced loading, BFR-LIX has been hypothesized to be a suitable candidate for combating postoperative sarcopenia and loss of function. As a result of occlusion, BFR-LIX elicits acute intramuscular hypoxia and an accumulation of lactate (local and systemic) with corresponding decreases in $\mathrm{pH}$ and increased in $\mathrm{CO}_{2}{ }^{43-45}$ These responses have been observed to facilitate postexercise increases in anabolic signaling (centrally and locally) as well as significantly influence metabolic adaptations to exercise training. ${ }^{46-48}$

In addition to the production of metabolites such as lactate and $\mathrm{CO}_{2}$ into circulation during strenuous exercise, skeletal muscle has also been observed to release a host of myokines that have the capacity to act in an autocrine, paracrine, and endocrine manner. ${ }^{49-51}$ Cumulatively, it is hypothesized that immediately following the occlusive stimulus, the release of these effectors into circulation may stimulate anabolism (for muscle, bone, and connective tissue) locally as well as systemically. ${ }^{21}$ For example, BFR-LIX has been observed to acutely increase systemic growth hormone $(\mathrm{GH})^{29,30,50,52}$ release similar to high-intensity exercise in response to elevations in systemic lactate concentrations among other central mechanisms. ${ }^{53,54}$ Although there is ongoing debate as to the direct role that GH may have with regard to skeletal muscle responses to exercise, ${ }^{31}$ upregulation and release of systemic IGF-1 (a potent stimulator of mammalian target of rapamycin complex 1 -mediated anabolism) as a result of GH signaling at the liver has been previously attributed to findings of both Abe et al. ${ }^{55}$ and Takano 
et al., ${ }^{56}$ who observed chronic and acute increases in systemic IGF-1 following BFR-LIX training. Notably, both GH and IFGs have been shown to play an important role in satellite cell proliferation and differentiation during recovery. ${ }^{57-59}$ Therefore, because of the multinucleated nature of skeletal muscle, BFR may indirectly yield chronic training responses via increased potential for future growth in response to exercise. ${ }^{21,31}$ These systemic signaling mechanisms have been postulated to have impact in both the occluded limb undergoing BFR as well as tissues proximal to the site of occlusion (discussion to follow). ${ }^{21}$

In addition to local and systemic impacts on muscle, recent pilot data indicate that BFR-LIX may have a positive impact on bone and possibly connective tissue. ${ }^{11}$ For example, it was recently observed that during 12 weeks of rehabilitation from anterior cruciate ligament (ACL) reconstruction, the incorporation of BFRLIX yielded significant reductions in whole limb and site-specific bone loss compared with standard rehabilitation alone. ${ }^{11}$ It has been hypothesized that venous occlusion may lead to fluid shifts causing increased intramedullary pressure and interstitial fluid flow within the bone. ${ }^{60}$ Previous observations also suggest that the response of bone to chronic exercise is potentially interlinked with skeletal muscle with regards to mechanical, systemic, and local signaling factors. ${ }^{44-46}$ Relatedly, myokines secreted from muscle during exercise in an intensity/stress dependent manner are known to act on bone metabolism in either proformation or resorption capacities. ${ }^{51,61-64}$ In addition, both systemic and muscle-derived IGFs and fibroblast growth factors are also known to act directly at the muscle-bone interface. ${ }^{62,65}$ Lastly, BFR has been previously observed to inhibit myostatin (a negative regulator of muscle and bone anabolism) expression. ${ }^{21,31,66}$ Importantly, inhibition of myostatin action and/or expression has been observed to improve muscle and fracture healing following trauma. ${ }^{60,66}$ While further research will be required to determine cause and effect, the present data provide impactful evidence that BFR may be a suitable tool for combatting postoperative bone loss and potentially assisting with bone graft integration at the knee.

\section{Skeletal Muscle Activation}

The overall anabolic response to resistance exercise is largely governed by the volume of work performed. This can be quantified as the total mechanical work (total sets $\times$ repetitions performed), the total metabolic cost of the exercise bout, and the amount of muscle recruited for a given exercise or set of exercises. However, when comparing BFR-LIX with standard LIX performed to fatigue, electromyographic (EMG) studies (used an indirect indicator of skeletal muscle activation) have resulted in mixed findings. For example,
Wernbom et al. ${ }^{67}$ observed no difference in peak muscle activation (expressed as EMG amplitude; EMGa) during a lower-extremity fatigue protocol comparing BFR-LIX with LIX alone. In an upperextremity model, Counts et al. ${ }^{68}$ observed no difference in EMGa when performing the same exercise under differing occlusion pressures ranging from $40 \%$ to $90 \%$ limb occlusion pressure (LOP). However, in a similar upper-extremity model evaluating fatiguing triceps extensions and biceps curls, Yasuda et al. ${ }^{44}$ observed increases in EMGa with increasing repetitions using the standard 30-15-15-15 protocol. Fatela et al. ${ }^{69}$ observed increased EMGa in the rectus femorus and vastus lateralis during leg extension exercise with increasing occlusion pressure ranging from $40 \%$ to $80 \%$ LOP. Lastly, although frequently described as being similar to high-intensity exercise, Bordessa et al. ${ }^{70}$ observed greater EMGa with high intensity leg extension exercise $(80 \%$ l-repetition maximum) compared with low intensity (30\% l-repetition maximum) with BFR. Using a similar exercise protocol, Teixeira et al. ${ }^{71}$ recently observed that during high-intensity leg extension exercise, the application of BFR reduced EMGa but did increase markers of metabolic stress, a finding similar to Dankel et al., ${ }^{72}$ who observed no additive effect of BFR during high-intensity elbow flexion exercise. In summary, the degree to which BFRLIX impacts muscle recruitment distal to the cuff requires further study. With regard to high-intensity exercise, BFR does not appear to elicit an additive effect indicating an upper threshold related to exercise intensity.

\section{Proximal Effects of BFR}

Previous reports characterizing the local and systemic responses of BFR-LIX have increased interest in the potential proximal benefits of occlusion training. This includes responses to tissues directly proximal to the site of the occlusion, responses in the contralateral limb relative to the limb undergoing BFR-LIX, and wholebody systemic responses. Recent investigations are now beginning to provide critical insight on the efficacy of BFR for tissues located proximally to the occlusion site.

\section{Effects of BFR on Skeletal Muscle Directly Proximal to the Occlusion Site}

Until recent years, many of the potential benefits of BFR were thought to be limited to tissues undergoing occlusion (distal to where the pressure is applied around the contracting limb). However, recent findings indicate that there may be some benefit to tissues directly proximal to the occlusion site. Although the mechanisms of action are still under a great deal of investigation, such findings have important clinical implications for the utility of BFR. In a recent study, 
Lambert et al. ${ }^{12}$ observed that following 8 weeks of low load bilateral rotator cuff training for the shoulder, BFR-LIX (performed under 50\% LOP) yielded greater increases in whole limb and shoulder region muscle mass, greater improvements in muscular work capacity compared, and some greater improvements in isometric strength compared with LIX alone in healthy. untrained adults. In this study, participants performed 4 common rotator cuff strengthening exercises (cable internal rotation, cable external rotation, dumbbell scaption, and side-lying dumbbell external rotation) performing an initial set of 30 repetitions followed 2 sets of 15 repetitions and a final set performed to fatigue (trained twice per week). These findings were paired with greater EMGa observed during fatigue testing while limbs were undergoing occlusion compared to unoccluded. ${ }^{12}$ These results are somewhat in line with previous findings that BFR may result in greater proximal muscle activation as a result of occlusion-induced distal fatigue. ${ }^{15,19,73}$ For example, Yasuda et al. ${ }^{73}$ were among the first to propose this after observing greater pectoralis muscle activation paired with improvements in muscle size in strength following BFR-LIX training during bench press exercise. However, in contrast to the hypothesis that increases in EMGa would result from prefatiguing of occluded distal musculature, Lambert et al. ${ }^{12}$ observed increased EMGa at the onset of exercise rather than after a series of repetitions. Therefore, it was hypothesized that these responses may have resulted, in part, from proprioceptive reflex mechanisms involved in detecting stretch and changes pressure (applied by the arm cuff) within muscle that may affect various chains of movement at the shoulder. ${ }^{74-76}$ Regarding improvements in muscle mass and strength, Bowman et al. ${ }^{13}$ also observed increased limb circumference and strength gains after 6 weeks of BFR-LIX training for the rotator cuff using a similar training protocol to Lambert et al. ${ }^{12}$ However, Brumitt et al. ${ }^{77}$ observed no difference between BFR-LIX and LIX alone with regards to strength or muscle thickness following twice weekly training of side-lying dumbbell external rotation. Therefore, it likely that there is a volume threshold or cumulative time under occlusion may be required to elicit proximal beneficial responses. This may require either greater exercise volumes for a given exercise or the inclusion of multiple, related exercises to elicit a desired response. Similar to the aforementioned findings with regards to upperextremity exercise, Bowman et al. ${ }^{14}$ observed greater improvements in lower-extremity strength, endurance, and hypertrophy with 6 weeks of BFR-LIX compared with LIX alone. These findings were in contrast to Sakamaki et al., ${ }^{78}$ who examined the proximal and distal adaptations to BFR during chronic walk training. The contrast between these 2 investigations suggests that similar to current upperextremity findings, there may be intensity and volume thresholds required for beneficial proximal responses to occur.

\section{Potential Systemic Mechanisms That May Influence Proximal Responses to BFR}

In addition to the potential mechanisms discussed with regard to muscle activation of tissues directly proximal to the site of occlusion, systemic anabolic signaling mechanisms (previously described) also have been hypothesized to play a role in the proximal response to BFR-LIX for muscle directly proximal to the site of occlusion, contralateral effects, and whole body cross-over effects. ${ }^{13-15,21,31}$ However, data on the direct measurement of these potential effects are extremely limited and, therefore, most current conclusions related to systemic action have been derived from indirect observations. For example, Bowman et al. ${ }^{13,14}$ observed increased grip strength measures in the contralateral limb to the BFR treatment limb following rotator cuff training and, in a separate study, observed increased thigh girth and knee extension strength in the contralateral limb to the BFR treatment limb following lower-extremity resistance exercise. Although no direct local or systemic measures of anabolic signaling were made, it was inferred that these responses may have been, in part, to systemic anabolic signaling mechanisms. Future mechanistic studies will be required to determine the degree to which systemic signaling mechanisms during and following BFR-LIX may impact tissues not subjected to occlusion. Furthermore, as systemic responses to resistance training are largely governed by training volume, work to rest intervals, metabolic cost of exercise, exercise intensity, and the amount muscle engaged in contractile activity, several factors remain unknown with regard to thresholds for the stimulus needed for a given systemic effect to determine if the use of BFR is practical for a given desired outcome. In the case of BFR-LIX, these factors included, but are not limited to, (1) bilateral versus contralateral training; (2) small muscle single joint versus large muscle multijoint exercises; (3) the total amount of exercises performed; (4) total time under occlusion; and (5) upper- versus lower-extremity exercises using considerably different quantities of contractile tissue. For example, fatiguing bilateral leg press under BFR would likely result in differential systemic responses compared to unilateral rotator cuff exercise.

Although several aspects of BFR remain to be investigated, the following findings provide some support (summarized in Fig 1) for its use for clinical application for treatment and preventative training for beneficial proximal outcomes. 


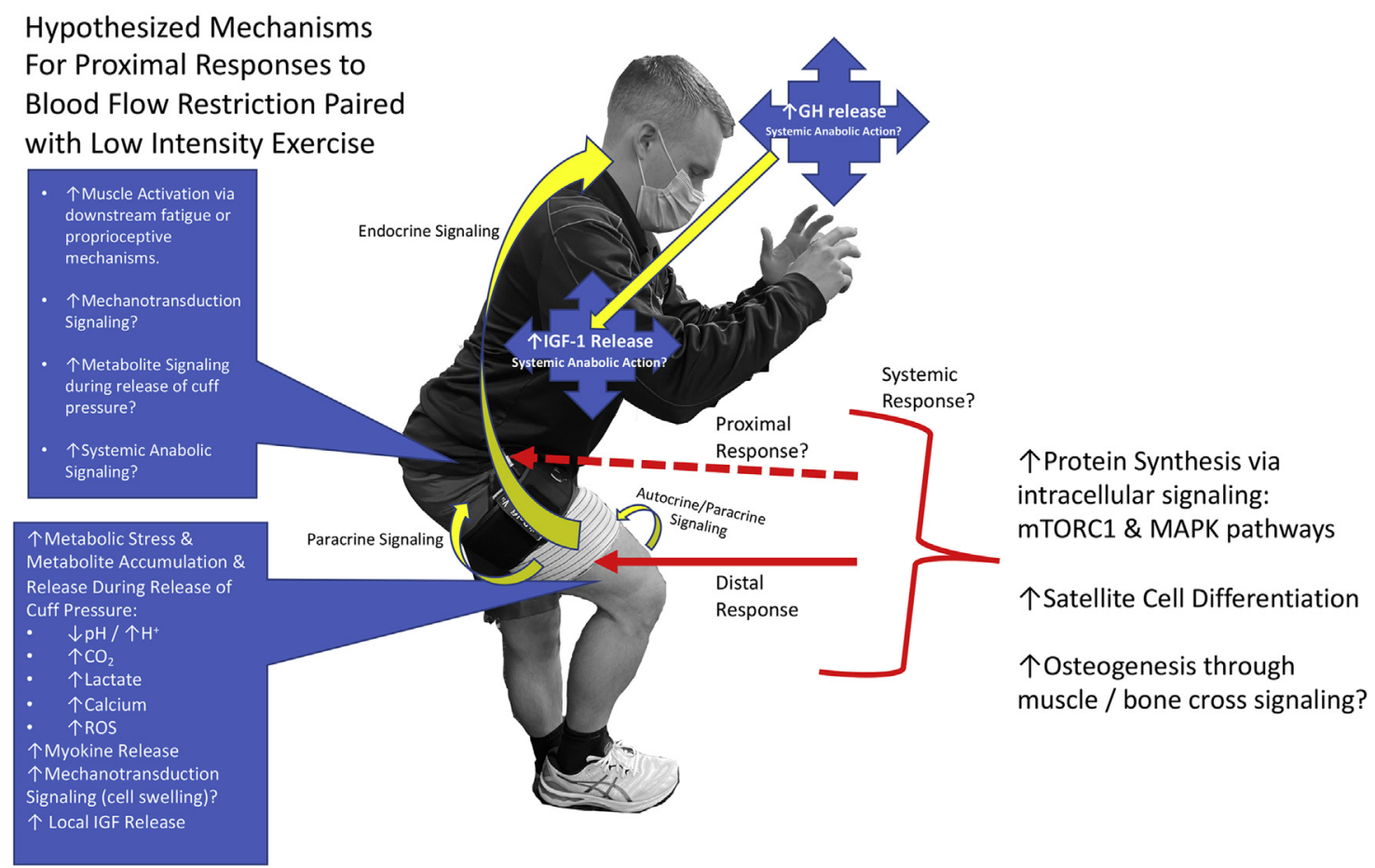

Fig 1. Potential mechanisms for stimulating proximal effects during blood flow restriction training paired with low-intensity resistance exercise. (GH, growth hormone; IGF, insulin-like growth factor; MAPK, mitogen-activated protein kinase; mTORC1, mammalian target of rapamycin complex 1; ROS, reactive oxygen species.)

\section{Current Paradigms in Clinical Use}

\section{Initial Findings in Distal Musculature}

In early publications on BFR with exercise, naturally, the largest muscles distal to the occlusive device receive a majority of the attention. The quadriceps is one particular muscle group popular for research due to its size and dominance in functional lower extremity activities. ${ }^{5,6,10,11}$ An early study by Eiken et al. ${ }^{79}$ examined the effect of "ischemic training" on quadriceps femoris muscle activity during a single-leg cycling trial. They found that blood flow-restricted training induces "...an increase in the share of the muscle cross-sectional area..." indicating the potential for ischemically induced changes in the metabolic characteristics of the muscle. ${ }^{79}$ These findings were likely instrumental in setting the stage for further study into quadriceps function with BFR nearly two decades later. Cook et al. ${ }^{2}$ found that lower loads could be used with knee extensions and BFR to elicit a similar fatigue effect in the quadriceps versus high-load exercise. The potential for significant muscle strength gains (or at least atrophy mitigation) incites significant attention from physical therapists as rehabilitation protocols are typically designed around these facets. More recent work, including a systematic review with meta-analysis from Van Cant et al., ${ }^{80}$ build on the premises from authors previous, indicating that "BFR could be a useful option for patients with knee conditions where conventional quadriceps strengthening program exacerbate knee symptoms." Undoubtedly, the findings from these studies are promising to rehabilitation professionals, especially when considering quadriceps activity and knee function. However, a common cautionary plea in the early literature indicates a need for further research into pathologic individuals, especially those involved in postsurgical cases.

\section{Postsurgical Potential}

Postoperatively, patients encounter significant muscle loss due to disuse atrophy, traumatic inhibition, and edema. ${ }^{6,11,81}$ This "muscle dump" coincides with significant strength and function impairments that often take months to years to regain. ${ }^{6}$ BFR has taken a recent precedent in the literature involving postsurgical cases and the subsequent recovery and rehabilitation. ACL reconstruction involves prominent quadriceps weakness following surgery, greatly limiting patients in the acute stages of rehab. ${ }^{5}$ Furthermore, suboptimal function of the knee extensors can lead to an unsuccessful return to sport, reinjury, or the development of knee arthritis later in life. ${ }^{82-84}$ To mitigate the postsurgical atrophy seen in patients with ACL injury, physical therapists recently have seen significant improvements in the early phases of rehabilitation with the use of BFR. ${ }^{11,85}$ Typically, patients are performing low-level exercises early in the rehabilitation process (such as quadriceps or 
Table 1. Recommended Strengthening Parameters

\begin{tabular}{|c|c|c|}
\hline \multicolumn{3}{|c|}{ Clinical Recommendations for Strengthening ${ }^{1,4,15,22}$} \\
\hline & Upper & \\
\hline & Extremity & Lower Extremity \\
\hline Occlusive pressure & $50 \%$ LOP & $80 \%$ LOP \\
\hline Resistance/intensity & $\begin{array}{l}30 \%-50 \% 1 \mathrm{RM}, \\
\text { MVC }\end{array}$ & $\begin{array}{l}20 \%-30 \% 1 R M, \text { MVC } \\
\text { (7-8 RPE) }\end{array}$ \\
\hline Repetitions/sets & $\begin{array}{l}30 / 15 / 15 / 15,30 \\
\text { second rest-periods }\end{array}$ & $\begin{array}{l}\text { 30/15/15/15, } 30 \text { second } \\
\text { rest-periods }\end{array}$ \\
\hline Frequency & $2-3 \times / \mathrm{wk}$ & $2-3 \times / \mathrm{wk}$ \\
\hline Duration & $8+w k$ & $8-12 \mathrm{wk}$ \\
\hline
\end{tabular}

lRM, 1-repetition maximum; LOP, limb occlusion pressure; MVC, maximal voluntary contraction; RPE, rating of perceived exertion.

hamstrings isometrics) in an effort to regain neuromuscular control and activation. ${ }^{5}$ Furthermore, some surgical protocols (especially those involving cartilaginous interventions) require significant periods of partial to complete reduction of weight-bearing through the limb. ${ }^{25,86}$ Several studies have observed positive benefits in exercises involving limited weightbearing scenarios, which hold promise to be advantageous for patient progress and function. ${ }^{25,86-88}$ Similar to ACL reconstruction and other lower-extremity orthopaedic surgery, total joint replacement in the lower extremity lends to significant functional decline and impairment. ${ }^{89-93}$ Total knee and total hip arthroplasty are becoming more popular for the aging population. Individuals frequently suffer up to an $80 \%$ loss in knee-extension strength in the initial days to weeks following surgery. ${ }^{89-91}$ Generally, the trauma from the surgery is responsible for the pain and diminished function, but most patients can perform light exercise and bear weight through the limb within 24 hours postoperatively. ${ }^{92,93}$ Currently, randomized controlled trials with BFR for individuals following an arthroplasty are limited and require further research. The lower-intensity nature of BFR and exercise may be enticing for this population in the future.

\section{Aerobic Considerations}

While this review has discussed important considerations for muscular strength, we must also consider recent introspect into the antithetical realm-aerobic capacity. Greater degrees of aerobic fitness have been shown to improve sports performance, increase rehabilitation potential, and improve general health. ${ }^{94-96}$ Similarly to strength training, improving aerobic performance requires significant training at a high threshold. Often, individuals must incorporate activities which place them at or above $70 \%$ of their maximal oxygen consumption $\left(\mathrm{VO}_{2} \mathrm{max}\right)$ to achieve a successful training effect. ${ }^{97,98}$ Unfortunately, many circumstances, such as poor health, injury, surgery, lack of time, and/ or resources, can hinder one's ability to incorporate aerobic activities into their regimen. ${ }^{99,100}$ Interestingly, BFR seems to have found its way into the aerobic environment, establishing similar positive effects seen in anaerobic training. Bennett and Slattery ${ }^{101}$ performed a systematic review on the effects of BFR on aerobic capacity and performing exercises such as continuous cycling, treadmill walking, and interval training. Interestingly, they highlighted that 8 of the 11 studies indicated positive improvements in $\mathrm{VO}_{2} \max$ or $\mathrm{VO}_{2}$ peak. ${ }^{101}$ The studies that did not find significant benefits included older subjects or used much lower occlusive pressures. ${ }^{101}$ The modality of exercise does not seem to influence the observed changes in aerobic capacity.

\section{Pain Modulation}

Pain is a significant limiting factor for patients of all backgrounds and pathologies when in rehabilitation. Physical therapy interventions, inclusive of exercise, are often directly targeted at pain signaling due to acute injury, chronic musculoskeletal disorders, and postsurgical effects. Moderate-intensity exercise has been shown to actually induce an analgesic effect. ${ }^{8-10,102}$ However, if an individual suffers from pain during exercise, this can limit their productivity, reducing the potential to achieve exercise-induced hypoalgesia. ${ }^{8-10.102}$ Otherwise, therapeutic activities often are structured in an effort to avoid pain or increased discomfort. Korakakis et al. ${ }^{10}$ found clinically significant reductions in anterior knee pain for a sample of patients after low-level exercise with BFR. They found that the reduction in pain was relatively long-lasting as well (>45 minutes), which allowed for greater compliance and consistency with physical therapy activities. ${ }^{10}$ Various mechanisms behind this analgesic effect have been explored, including opioid and endocannabinoid-mediated pain inhibition, conditioned pain modulation, recruitment of high threshold motor units, exercise-induced metabolite production, and an interaction between cardiovascular and pain regulatory systems. ${ }^{8}$ Song et al. ${ }^{102}$ performed a systematic review of similar studies that examined these theoretical mechanisms and concluded that exercise with BFR may serve as an effective method of pain management for those who cannot train with higher loads. However, "...the roles of these mechanisms are still unclear and require further clarification."

\section{Neurologic/Systemic Disorders}

Orthopaedic pathology and procedures naturally hold a strong spotlight in the literature and clinical use of BFR with exercise. We often think of rehabilitation of muscle, bone, and connective tissue disorders as a relatively straightforward continuum with predictable outcomes and time frames. However, muscle 
Table 2. Exercise Selection for Strengthening

\begin{tabular}{|c|c|c|c|}
\hline \multicolumn{4}{|c|}{ Therapeutic Exercise Selection for Strengthening ${ }^{1,11-14}$} \\
\hline Muscle Group & Exercise & Muscle Group & Exercise \\
\hline $\begin{array}{l}\text { Periscapular } \\
\text { musculature }\end{array}$ & $\begin{array}{l}\text { - Resisted rows } \\
\text { - Shoulder extension } \\
\text { - I's/T's/Y's } \\
\text { - Serratus walk-ups } \\
\text { - Resisted shoulder retraction } \\
\\
\text { with external rotation }\end{array}$ & Gluteus medius/minimus & $\begin{array}{l}\text { - Sidelying straight leg raise } \\
\text { - Clamshells } \\
\text { - Quadruped fire hydrants } \\
\text { - Lateral band walks } \\
\text { - Standing hip abduction } \\
\text { - Lateral step ups }\end{array}$ \\
\hline
\end{tabular}

weakness, pain, and dysfunction are also hallmarks of certain neurologic or autoimmune diseases as well. For these individuals, similar rehabilitation principles hold true-build strength and mitigate atrophy/weakness as much as possible. Some notable studies have already set the groundwork for the use of BFR in patient populations outside of the orthopedic and sports realms. Liang et al. ${ }^{103}$ and Jørgensen et al. ${ }^{104}$ have performed similar randomized control trials in patient populations with sporadic inclusion-body myositis. Both groups examined similar variables in their patients-quality of life, strength, self-reported function, and endurance. Liang et al. ${ }^{103}$ found favorable results for the BFR group in all variables. Jørgensen et al. ${ }^{104}$ did not note significant differences in selfreported or objective outcomes, but they did find that the BFR group did not encounter diminished strength versus the controls. Thus, concluding that BFR with exercise may have a "preventive (retaining) effect on the disease-related decline in leg muscle strength, which may aid the long-term preservation of physical function and postpone the need for healthcare assistance." ${ }^{104}$ Furthermore, Mattar et al. ${ }^{105}$ found favorable results in quality of life, muscle strength, and function by using BFR and exercise with patients diagnosed with polymyositis and dermatomyositis. Muscle mass was also improved in their BFR group, which may indicate added benefit for these groups. ${ }^{105}$ Finally, Douris et al. ${ }^{106}$ studied BFR with exercise in an individual with Parkinson disease. Their outcomes were positive for improvements in strength and function as well as Parkinson-related outcome measures for quality of life. ${ }^{106}$ While a litany of research is becoming available in the orthopedic literature, the future of BFR may expand into a number of other populations as well.

\section{The Future of BFR: Proximal Potential}

Previous mechanistic models for BFR with exercise have favored distal structures and muscle groups over proximal regions such as the shoulder and hip. As further research is conducted, we are gaining a better perspective on how BFR may benefit a wider variety of clinical cases and the population.

The shoulder is a complex structure which requires a delicate balance between large and small muscles to establish a force couple of the scapulothoracic and glenohumeral architecture. ${ }^{107}$ This provides a proximally stable foundation for a distally mobile limb to perform unencumbered tasks such as reaching, pushing, pulling, or throwing. ${ }^{107,108}$ The upper extremity is typically active in both open- and closed-chain scenarios, requiring notable strength and proprioception to avoid injury. In physical therapy and rehabilitation, careful consideration is warranted to restore function to the shoulder region and usually consists of meticulous strengthening exercises using progressive loading. ${ }^{107,108}$ The inclusion of BFR with lower loads for the shoulder and associated structures is of particular interest in an effort to potentially accelerate rehabilitation, minimize adverse effects of postsurgical conditions, and to potentially prevent injury to a vulnerable region. Unfortunately, few studies exist examining the effect of BFR on the proximal muscles including the rotator cuff and periscapular musculature. As mentioned previously, Yasuda et al. ${ }^{73}$ first elucidated the positive effects of BFR for the pectoralis major following 8 weeks of bench press training. Lambert et al. ${ }^{12}$ performed a randomized-controlled trial with multiple low-intensity exercises for the rotator cuff, observing strength, endurance, muscle activity, and lean-mass changes between groups either using BFR or 
Table 3. Recommended Aerobic Parameters

\begin{tabular}{ll}
\hline \multicolumn{2}{c}{ Clinical Recommendations for Aerobic Activities } \\
\hline Occlusive pressure & $50 \%-80 \% \mathrm{LOP}$ \\
Resistance/intensity & $30 \%-40 \% \mathrm{VO}_{2} \mathrm{max}$ \\
Time & $10-30 \mathrm{~min}$ \\
Frequency & $3-4 \times /$ week \\
Duration & $6-8+$ weeks \\
\hline
\end{tabular}

LOP, limb occlusion pressure; $\mathrm{VO}_{2} \max$, maximal oxygen consumption.

not. Dankel et al. ${ }^{15}$ found similar results for proximal and distal muscles in the upper extremity, noting that the inability to directly occlude certain muscles does not limit their potential to achieve strength gains at lower loads.

Similar to the shoulder, the hip remains a vital region to the active population. A prominent weight-bearing structure, the femoroacetabular joint and adjoining musculature are highly active with many functional movements and sports-related activities. ${ }^{17}$ The gluteus maximus, medius, and minimus each provide a unique role in establishing a powerful, yet stable synergy for high performance mobility. ${ }^{17}$ Furthermore, the hip serves as a crucial component to the lower-extremity kinetic chain as deficits in hip strength, power, or endurance are predisposing factors for knee, ankle, or foot injuries. ${ }^{17}$ The role of BFR in lower-extremity rehabilitation has been well-documented, but primarily for strength and function of muscles distal to the occlusion stimulus. The hip seems to hold significant potential in this realm as weight-bearing is often limited following severe injury or surgery and the resultant muscle atrophy and weakness is profound. ${ }^{90}$ However, direct study for the hip musculature is severely understudied at this time. Bowman et al. ${ }^{14}$ again examined proximal, distal, and contralateral limb strength and circumference with randomized groups exercising with (or without) BFR. Similar to their upper-extremity results, the data favor BFR with lower-extremity exercise, even proximally at the hip. ${ }^{14}$ As of this review, no other published data exist for the gluteal or intrinsic hip musculature with BFR and exercise.

\section{Clinical Utility and Practice: From Bench to Bedside}

Conceptual and mechanistic models for the clinical use of BFR have been widely discussed and studied over the past decade. Many articles have a focal intent in their research (strengthening versus aerobic fitness vs hormonal/systemic responses) but provide a multitude of methods or techniques with no one globallyaccepted protocol available. However, recent systematic reviews and meta-analyses are progressively merging similar findings to provide clinicians with greater consistency in clinical application. ${ }^{1,4,15,22,101,109}$
Table 1 indicates current clinical recommendations for strength training with BFR based on this review. Currently unknown are how differentiation in these protocols affect proximal versus distal musculature to a greater degree. Table 2 includes selected activities prevalent in the literature and clinical practice targeted at vital proximal musculature that take precedence in upper- or lower-extremity rehabilitation. To date, no studies have examined EMG activity of the lower extremity proximal musculature during the proposed exercises. Lambert et al. ${ }^{12}$ are the first to elucidate EMG signaling of the proximal musculature of the upper extremity during BFR with exercise targeted at the rotator cuff over an 8-week interventional design.

In addition to strengthening, aerobic activities remain commonplace in rehabilitation and physical therapy dependent on patient goals and needs. Various trials have been performed with BFR and aerobic exercise, generally indicating that this combination produces improvements in aerobic performance and fitness in various populations irrespective of training intensity. ${ }^{101,109}$ Interestingly, there may be differences in observed adaptations based on age, however. ${ }^{101}$ Table 3 outlines current clinical recommendations for aerobic activities with BFR. Note that BFR with upperextremity aerobic activities (arm ergometer, swimming) is vastly understudied.

Finally, a relatively newer method of using BFR for the benefit of patient care is known as ischemic preconditioning (IPC). The concept of IPC has been observed throughout the medical literature for a number of years and can be defined as the exposure to brief periods of circulatory occlusion and reperfusion to protect local or systemic organs against subsequent bouts of ischemia. ${ }^{110}$ Furthermore, we have seen studies find an improvement in $\mathrm{VO}_{2} \mathrm{max}$, increased sports performance, and increased strength/endurance. ${ }^{10-115}$ Franz et al. ${ }^{116}$ also found that IPC may blunt exercise-induced muscle damage when performed before bouts of eccentric exercise of the muscle flexors. Tanaka et al. ${ }^{117}$ theorize that the origin of the beneficial effects from IPC may likely be the enhancement of mitochondrial metabolism in skeletal muscle. Naturally, the occlusive stimulus used with BFR makes sense when considering the potential systemic and local effects of IPC. Typically, most protocols involve full occlusion without the addition of exercise. Table 4 provides a current guideline for IPC of either the upper or lower extremities.

\section{Injury Prevention and Recovery}

Sports participation in the United States is constantly growing and evolving. Professional sports account for a multibillion-dollar industry and consist of some of the highest skilled athletes in the world. With some athletes now signing contracts for several hundred million 
Table 4. Recommended Ischemic Preconditioning (IPC) Parameters

\begin{tabular}{lc}
\hline \multicolumn{1}{c}{ Clinical Recommendations for IPC ${ }^{110}$} \\
\hline Occlusive pressure & $100 \%$ \\
Exercise & None, static \\
Time/duration & $5 \mathrm{~min}$ of occlusion \\
& $5 \mathrm{~min}$ break \\
Sets & $3-4$ \\
Frequency & $3-5 \times / \mathrm{wk}$ \\
Duration & $6-8 \mathrm{wk}$ \\
\hline
\end{tabular}

dollars (USD), the science that goes into their performance is extremely complex and vital for teams to see a return on their investment. Immense research has been performed on performance optimization and many studies will allude to a delicate balance of training and recovery. The ability to reduce physical and mental stress while remaining productive in training regimens plays a key role in an athlete or teams' success. The potential for BFR to meet these needs might be the "holy grail" for future performance research.

The sport of basketball is an international sensation, widely popular with tall and athletic individuals in an extremely competitive environment. A vast majority of injuries encountered in the sport are about the lower extremity and can be directly correlated to fatigue and intensity. Several researchers have studied the effect of competitive basketball games on fatigue levels and most indicate a cumulative workload effect, especially with multiple games played per week. ${ }^{118-121}$ Furthermore, Rubin et al. ${ }^{118}$ detected significant seasonal changes in the articular cartilage of basketball players and regional differences in the articular cartilage that are indicative of basketball-specific stress on the femoral cartilage. Thus, maintaining adequate muscle mass and strength about the lower extremities can be particularly challenging for the basketball player, especially in a full 82game season as played in the National Basketball Association. Players experience even more demand in playoff situations with congested schedules (games every other day) when in need of their absolute peak performance. ${ }^{121}$ Using BFR with lower loads and mitigating loss of Type II muscle would be highly beneficial for basketball athletes. Additionally, Faltus et al. ${ }^{122}$ provide further theoretical concepts for BFR within the basketball population, inclusive of chronic pain management, increased motor unit recruitment, and kinetic chain/proximal improvements. Further study is needed within these athletes to determine appropriate loading parameters as well as exercise selection and frequency.

Baseball is another sport with extremely long and stressful competitive seasons. Major League Baseball players are subjected to 162 games in a regular season with multiple series in playoff rounds. These athletes encounter significant stress/strain over the course of competition, producing some of the highest angular velocities recorded at the shoulder and elbow while throwing at maximum intensity. Khalil et al. ${ }^{123}$ and Chalmers et al. ${ }^{124}$ discovered anatomical changes about the shoulder and elbow that occur in the baseball athlete during a long season. More specifically, the ulnar collateral ligament exhibits a thickening effect, potentially in relation to a protective response to the large amounts of valgus stress in the elbow during throwing. ${ }^{124}$ Furthermore, significant decreases in range of motion are common, especially in the shoulder, towards the end of the season. ${ }^{124,125}$ Therefore, to protect from injury or time lost, baseball athletes need to maintain optimal strength without adversely affecting performance or tissue quality. BFR has been shown to improve strength, endurance, and lean mass about the upper extremity with relatively low loads which would be particularly helpful for the baseball athlete. ${ }^{12}$ With future study toward optimal training loads to performance enhancement, we can better understand how the role of BFR may expand in the sport of baseball, especially for proximal musculature such as the shoulder.

Ultimately, professional and amateur sports can place large demands on the human body. Athletic trainers, physical therapists, and performance specialists are tasked with the role of helping athletes maintain peak performance while mitigating injury risk. The inclusion of BFR regimens seems to hold promise, especially for proximal musculature due to the inherent low loads utilized during training.

\section{Conclusions}

In conclusion, the combination of BFR-LIX seems to promote an improvement in skeletal muscle anabolism at low loads to mitigate atrophy postinjury or surgery and improve strength while minimizing risk. Interestingly, benefits have been found in musculature proximal to the occlusive stimulus that may lend promise to a greater variety of patient populations and conditions. Furthermore, an increasing demand for BFR use in the sports world warrants further research for performance research and recovery.

\section{References}

1. Hughes L, Paton B, Rosenblatt B, Gissane C, Patterson SD. Blood flow restriction training in clinical musculoskeletal rehabilitation: A systematic review and meta-analysis. Br J Sports Med 2017;51:1003-1011.

2. Cook SB, Clark BC, Ploutz-Snyder LL. Effects of exercise load and blood-flow restriction on skeletal muscle function. Med Sci Sports Exerc 2007;39:1708-1713.

3. Loenneke JP, Wilson JM, Marín PJ, Zourdos MC, Bemben MG. Low intensity blood flow restriction 
training: A meta-analysis. Eur J Appl Physiol 2012;112: 1849-1859.

4. Scott BR, Loenneke JP, Slattery KM, Dascombe BJ. Exercise with blood flow restriction: an updated evidencebased approach for enhanced muscular development. Sports Med 2015;45:313-325.

5. van Melick N, van Cingel RE, Brooijmans F, et al. Evidence-based clinical practice update: practice guidelines for anterior cruciate ligament rehabilitation based on a systematic review and multidisciplinary consensus. $\mathrm{Br} J$ Sports Med 2016;50:1506-1515.

6. Lepley LK, Davi SM, Burland JP, Lepley AS. Muscle atrophy after ACL injury: Implications for clinical practice. Sports Health 2020;12:579-586.

7. Cook SB, Murphy BG, Labarbera KE. Neuromuscular function after a bout of low-load blood flow-restricted exercise. Med Sci Sports Exerc 2013;45:67-74.

8. Hughes L, Patterson SD. Low intensity blood flow restriction exercise: Rationale for a hypoalgesia effect. Med Hypotheses 2019;132:109370.

9. Hughes L, Patterson SD. The effect of blood flow restriction exercise on exercise-induced hypoalgesia and endogenous opioid and endocannabinoid mechanisms of pain modulation. J Appl Physiol (1985) 2020;128:914-924.

10. Korakakis V, Whiteley R, Epameinontidis K. Blood Flow Restriction induces hypoalgesia in recreationally active adult male anterior knee pain patients allowing therapeutic exercise loading. Phys Ther Sport 2018;32:235-243.

11. Lambert B, Hedt CA, Jack RA, et al. Blood flow restriction therapy preserves whole limb bone and muscle following ACL reconstruction. Orthop J Sports Med 2019;7: 2325967119 S00196 (3 suppl 2).

12. Lambert B, Hedt C, Daum J, et al. Blood flow restriction training for the shoulder: A case for proximal benefit. Am J Sports Med 2021;49:2716-2728.

13. Bowman EN, Elshaar R, Milligan H, et al. Upper-extremity blood flow restriction: The proximal, distal, and contralateral effects-a randomized controlled trial. J Shoulder Elbow Surg 2020;29:1267-1274.

14. Bowman EN, Elshaar R, Milligan H, et al. Proximal, distal, and contralateral effects of blood flow restriction training on the lower extremities: A randomized controlled trial. Sports Health 2019;11:149-156.

15. Dankel SJ, Jessee MB, Abe T, Loenneke JP. The effects of blood flow restriction on upper-body musculature located distal and proximal to applied pressure. Sports Med 2016;46:23-33.

16. Escamilla RF, Andrews JR. Shoulder muscle recruitment patterns and related biomechanics during upper extremity sports. Sports Med 2009;39:569-590.

17. Bowman KF Jr, Fox J, Sekiya JK. A clinically relevant review of hip biomechanics. Arthroscopy 2010;26: 1118-1129.

18. Takarada Y, Sato Y, Ishii N. Effects of resistance exercise combined with vascular occlusion on muscle function in athletes. Eur J Appl Physiol 2002;86:308-314.

19. Takarada Y, Takazawa H, Sato Y, Takenoshita S, Tanaka Y, Ishii N. Effects of resistance exercise combined with moderate vascular occlusion on muscular function in humans. J Appl Physiol 2000;88:2097-2106.
20. Sundberg CJ. Exercise and training during graded leg ischaemia in healthy man with special reference to effects on skeletal muscle. Acta Physiol Scand Suppl 2004;615:1-50.

21. Lambert BS, Hedt C, Moreno M, Harris JD, McCulloch P. Blood flow restriction therapy for stimulating skeletal muscle growth: Practical considerations for maximizing recovery in clinical rehabilitation settings. Tech Orthop 2018;33:89-97.

22. Loenneke JP, Kim D, Fahs CA, et al. Effects of exercise with and without different degrees of blood flow restriction on torque and muscle activation. Muscle Nerve 2015;51:713-721.

23. Yamanaka T, Farley RS, Caputo JL. Occlusion training increases muscular strength in Division IA football players. J Strength Cond Res 2012;26:2523-2529.

24. Patterson SD, Hughes L, Warmington S, et al. Blood flow restriction exercise: Considerations of methodology, application, and safety. Front Physiol 2019;10:533.

25. Tennent DJ, Hylden CM, Johnson AE, Burns TC, Wilken JM, Owens JG. Blood flow restriction training after knee arthroscopy: A randomized controlled pilot study. Clin J Sport Med 2017;27:245-252.

26. Abe T, Loenneke JP, Fahs CA, Rossow LM, Thiebaud RS, Bemben MG. Exercise intensity and muscle hypertrophy in blood flow-restricted limbs and non-restricted muscles: A brief review. Clin Physiol Funct Imaging 2012;32: 247-252.

27. Centner C, Wiegel P, Gollhofer A, König D. Effects of blood flow restriction training on muscular strength and hypertrophy in older individuals: A systematic review and meta-analysis. Sports Med 2019;49:95-108.

28. Egan B, Zierath JR. Exercise metabolism and the molecular regulation of skeletal muscle adaptation. Cell Metab 2013;17:162-184.

29. Fry CS, Glynn EL, Drummond MJ, et al. Blood flow restriction exercise stimulates mTORCl signaling and muscle protein synthesis in older men. J Appl Physiol (1985) 2010;108:1199-1209.

30. Fujita S, Abe T, Drummond MJ, et al. Blood flow restriction during low-intensity resistance exercise increases S6Kl phosphorylation and muscle protein synthesis. J Appl Physiol (1985) 2007;103:903-910.

31. Hwang PS, Willoughby DS. Mechanisms behind blood flow-restricted training and its effect toward muscle growth. J Strength Cond Res 2019;33:S167-S179.

32. Shahbazian D, Roux PP, Mieulet V, et al. The mTOR/ PI3K and MAPK pathways converge on eIF4B to control its phosphorylation and activity. EMBO J 2006;25: 2781-2791.

33. Wortman RJ, Brown SM, Savage-Elliott I, Finley ZJ, Mulcahey MK. Blood flow restriction training for athletes: A systematic review. Am J Sports Med 2021;49: 1938-1944.

34. Loenneke J, Fahs C, Rossow L, Abe T, Bemben M. The anabolic benefits of venous blood flow restriction training may be induced by muscle cell swelling. Med Hypotheses 2012;78:151-154.

35. Rossi FE, De Freitas MC, Zanchi NE, Lira FS, Cholewa JM. The role of inflammation and immune cells 
in blood flow restriction training adaptation: A review. Front Physiol 2018;9:1376.

36. Gundermann DM, Walker DK, Reidy PT, et al. Activation of mTORCl signaling and protein synthesis in human muscle following blood flow restriction exercise is inhibited by rapamycin. Am J Physiol Endocrinol Metab 2014;306:E1 198-E1204.

37. Natsume T, Yoshihara T, Naito H. Electromyostimulation with blood flow restriction enhances activation of mTOR and MAPK signaling pathways in rat gastrocnemius muscles. Appl Physiol Nutr Metab 2019;44:637-644.

38. Coffey VG, Hawley JA. Concurrent exercise training: Do opposites distract? J Physiol 2017;595:2883-2896.

39. Fluckey JD, Knox M, Smith L, et al. Insulin-facilitated increase of muscle protein synthesis after resistance exercise involves a MAP kinase pathway. Am J Physiol Endocrinol Metab 2006;290:E1205-E1211.

40. Gao Y, Arfat Y, Wang H, Goswami N. Muscle atrophy induced by mechanical unloading: mechanisms and potential countermeasures. Front Physiol 2018;9:235.

41. McGlory C, Phillips SM. Exercise and the regulation of skeletal muscle hypertrophy. Prog Mol Biol Transl Sci 2015;135:153-173.

42. Baehr LM, West DW, Marshall AG, Marcotte GR, Baar K, Bodine SC. Muscle-specific and age-related changes in protein synthesis and protein degradation in response to hindlimb unloading in rats. J Appl Physiol 2017;122: 1336-1350.

43. Yasuda T, Abe T, Brechue WF, et al. Venous blood gas and metabolite response to low-intensity muscle contractions with external limb compression. Metabolism 2010;59:1510-1519.

44. Yasuda T, Fukumura K, Fukuda T, et al. Effects of lowintensity, elastic band resistance exercise combined with blood flow restriction on muscle activation. Scand J Med Sci Sports 2014;24:55-61.

45. Loenneke JP, Kim D, Fahs CA, et al. The influence of exercise load with and without different levels of blood flow restriction on acute changes in muscle thickness and lactate. Clin Physiol Funct Imaging 2017;37:734-740.

46. Jung W-S, Kim S-W, Kim J-W, Park H-Y. Resistance training in hypoxia as a new therapeutic modality for sarcopenia-a narrative review. Life 2021;11:106.

47. Moghetti P, Bacchi E, Brangani C, Donà S, Negri C. Metabolic effects of exercise. Sports Endocrinol 2016;47: 44-57.

48. Nalbandian M, Takeda M. Lactate as a signaling molecule that regulates exercise-induced adaptations. Biology 2016;5:38

49. Cornish SM, Bugera EM, Duhamel TA, Peeler JD, Anderson JE. A focused review of myokines as a potential contributor to muscle hypertrophy from resistance-based exercise. Eur J Appl Physiol 2020;120: 941-959.

50. Pearson SJ, Hussain SR. A review on the mechanisms of blood-flow restriction resistance training-induced muscle hypertrophy. Sports Med 2015;45:187-200.

51. Pedersen BK, Febbraio MA. Muscle as an endocrine organ: focus on muscle-derived interleukin-6. Physiol Rev 2008;88:1379-1406.
52. Loenneke J, Wilson G, Wilson J. A mechanistic approach to blood flow occlusion. Int J Sports Med 2010;31:1-4.

53. Godfrey RJ, Whyte G, Buckley J, Quinlivan R. The role of lactate in the exercise-induced human growth hormone response: Evidence from McArdle disease. Br J Sports Med 2009;43:521-525.

54. Sartorio A, Agosti F, De Col A, et al. Growth hormone and lactate responses induced by maximal isometric voluntary contractions and whole-body vibrations in healthy subjects. J Endocrinol Invest 2011 ; 34:216-221.

55. Abe T, Yasuda T, Midorikawa T, et al. Skeletal muscle size and circulating IGF-1 are increased after two weeks of twice daily "KAATSU" resistance training. Int J KAATSU Training Res 2005;1:6-12.

56. Takano H, Morita $\mathrm{T}$, Iida H, et al. Hemodynamic and hormonal responses to a short-term low-intensity resistance exercise with the reduction of muscle blood flow. Eur J Appl Physiol 2005;95:65-73.

57. Dumont NA, Bentzinger CF, Sincennes $\mathrm{M}-\mathrm{C}$, Rudnicki MA. Satellite cells and skeletal muscle regeneration. Compr Physiol 2015;5:1027-1059.

58. Schoenfeld BJ. Potential mechanisms for a role of metabolic stress in hypertrophic adaptations to resistance training. Sports Med 2013;43:179-194.

59. Duan C, Ren H, Gao S. Insulin-like growth factors (IGFs), IGF receptors, and IGF-binding proteins: Roles in skeletal muscle growth and differentiation. Gen Comp Endocrinol 2010;167:344-351.

60. Loenneke JP, Young KC, Fahs CA, Rossow LM, Bemben DA, Bemben MG. Blood flow restriction: rationale for improving bone. Med Hypotheses 2012;78: 523-527.

61. Hamrick MW. A role for myokines in muscle-bone interactions. Exerc Sport Sci Rev 2011;39:43.

62. Hamrick MW. The skeletal muscle secretome: An emerging player in muscle-bone crosstalk. BoneKEy Rep 2012;1.

63. Lang TF. The bone-muscle relationship in men and women. J Osteoporos 2011 1;2011:702735.

64. Walsh K. Adipokines, myokines and cardiovascular disease. Circ J 2009;73:13-18.

65. D'Amore PA, Brown RH Jr, Ku PT, et al. Elevated basic fibroblast growth factor in the serum of patients with Duchenne muscular dystrophy. Ann Neurol 1994;35: 362-365.

66. Elkasrawy M, Immel D, Wen X, Liu X, Liang L-F, Hamrick MW. Immunolocalization of myostatin (GDF-8) following musculoskeletal injury and the effects of exogenous myostatin on muscle and bone healing. J Histochem Cytochem 2012;60:22-30.

67. Wernbom M, Järrebring $R$, Andreasson MA, Augustsson J. Acute effects of blood flow restriction on muscle activity and endurance during fatiguing dynamic knee extensions at low load. J Strength Cond Res 2009;23: 2389-2395.

68. Counts BR, Dankel SJ, Barnett BE, et al. Influence of relative blood flow restriction pressure on muscle activation and muscle adaptation. Muscle Nerve 2016;53: 438-445.

69. Fatela P, Reis JF, Mendonca GV, Avela J, Mil-Homens P. Acute effects of exercise under different levels of blood- 
flow restriction on muscle activation and fatigue. Eur $J$ Appl Physiol 2016;1 16:985-995.

70. Bordessa JM, Hearn MC, Reinfeldt AE, et al. Comparison of blood flow restriction devices and their effect on quadriceps muscle activation. Phys Ther Sport 2021;49: 90-97.

71. Teixeira EL, Barroso R, Silva-Batista C, et al. Blood flow restriction increases metabolic stress but decreases muscle activation during high-load resistance exercise. Muscle Nerve 2018;57:107-111.

72. Dankel SJ, Buckner SL, Jessee MB, et al. Can blood flow restriction augment muscle activation during high-load training? Clin Physiol Funct Imaging 2018;38: 291-295.

73. Yasuda T, Fujita S, Ogasawara R, Sato Y, Abe T. Effects of low-intensity bench press training with restricted arm muscle blood flow on chest muscle hypertrophy: A pilot study. Clin Physiol Funct Imaging 2010;30:338-343.

74. Hollman JH, Berling TA, Crum EO, Miller KM, Simmons BT, Youdas JW. Do verbal and tactile cueing selectively alter gluteus maximus and hamstring recruitment during a supine bridging exercise in active females? A randomized controlled trial. J Sport Rehabil 2018;27:138-143.

75. Ploughman M, Shears J, Quinton S, et al. Therapists' cues influence lower limb muscle activation and kinematics during gait training in subacute stroke. Disabil Rehabil 2018;40:3156-3163.

76. Salles JI, Velasques B, Cossich V, et al. Strength training and shoulder proprioception. J Athletic Train 2015;50: 277-280.

77. Brumitt J, Hutchison MK, Kang D, et al. Blood flow restriction training for the rotator cuff: A randomized controlled trial. Int J Sports Physiol Perform 2020;15: 1175-1180.

78. Sakamaki M, Bemben MG, Abe T. Legs and trunk muscle hypertrophy following walk training with restricted leg muscle blood flow. J Sports Sci Med 2011;10:338.

79. Eiken O, Sundberg CJ, Esbjörnsson M, Nygren A, Kaijser L. Effects of ischaemic training on force development and fibre-type composition in human skeletal muscle. Clin Physiol 1991;11:41-49.

80. Van Cant J, Dawe-Coz A, Aoun E, Esculier JF. Quadriceps strengthening with blood flow restriction for the rehabilitation of patients with knee conditions: A systematic review with meta-analysis. J Back Musculoskelet Rehabil 2020;33:529-544.

81. Pourtaheri S, Issa K, Lord E, et al. Paraspinal muscle atrophy after lumbar spine surgery. Orthopedics 2016;39: e209-e214.

82. Lohmander LS, Ostenberg A, Englund M, et al. High prevalence of knee osteoarthritis, pain, and functional limitations in female soccer players twelve years after anterior cruciate ligament injury. Arthritis Rheum 2004;50:3145-3152.

83. Grindem H, Snyder-Mackler L, Moksnes H, et al. Simple decision rules can reduce reinjury risk by $84 \%$ after ACL reconstruction: The Delaware-Oslo ACL cohort study. $\mathrm{Br}$ J Sports Med 2016;50:804-808.

84. Beischer S, Gustavsson L, Senorski EH, et al. Young athletes who return to sport before 9 months after ACL reconstruction have a rate of new injury 7 times that of those who delay return. J Orthop Sports Phys Ther 2020;50:83-90.

85. Lu Y, Patel BH, Kym C, et al. Perioperative blood flow restriction rehabilitation in patients undergoing ACL reconstruction: A systematic review. Orthop J Sports Med 2020;8:2325967120906822.

86. Loenneke JP, Young KC, Wilson JM, Andersen JC. Rehabilitation of an osteochondral fracture using blood flow restricted exercise: A case review. J Bodyw Mov Ther 2013;17:42-45.

87. DePhillipo NN, Kennedy MI, Aman ZS, Bernhardson AS, O'Brien LT, LaPrade RF. The role of blood flow restriction therapy following knee surgery: Expert opinion. Arthroscopy 2018;34:2506-2510.

88. Kruithof EE, Thomas SA, Tripp P. Blood flow restriction therapy following microfracture surgery for osteochondritis dissecans in a collegiate athlete. Int $J$ Athletic Ther Training 2018;23:230-233.

89. Holm B, Kristensen MT, Bencke J, et al. Loss of kneeextension strength is related to knee swelling after total knee arthroplasty. Arch Physiol Med Rehabil 2010;91: 1770-1776.

90. Fukumoto Y, Ohata K, Tsukagoshi R, et al. Changes in hip and knee muscle strength in patients following total hip arthroplasty. J Jpn Phys Therap Assoc 2013;16:22-27.

91. Holm B, Kristensen MT, Husted H, et al. Thigh and knee circumference, knee-extension strength, and functional performance after fast-track total hip arthroplasty. $P M R$ 2011;3:117-124.

92. Holm B, Thorborg K, Husted H, et al. Surgery-induced changes and early recovery of hip-muscle strength, legpress power, and functional performance after fasttrack total hip arthroplasty: A prospective cohort study. PLoS One 2013;8:e62109.

93. Winther SB, Husby VS, Foss OA, et al. Muscular strength after total hip arthroplasty: A prospective comparison of 3 surgical approaches. Acta Orthop 2016;87:22-28.

94. Blair SN, Morris JN. Healthy hearts - and the universal benefits of being physically active: Physical activity and health. Ann Epidemiol 2009; 19:253-256.

95. Moore SC, Patel AV, Matthews CE, et al. Leisure time physical activity of moderate to vigorous intensity and mortality: A large pooled cohort analysis. PLoS Med 2012;9:e1001335.

96. Joyner MJ, Green DJ. Exercise protects the cardiovascular system: Effects beyond traditional risk factors. J Physiol 2009;587:5551-5558.

97. Astorino TA, Allen RP, Roberson DW, Jurancich M. Effect of high-intensity interval training on cardiovascular function, VO2max, and muscular force. J Strength Cond Res 2012;26:138-145.

98. Bacon AP, Carter RE, Ogle EA, Joyner MJ. VO2max trainability and high intensity interval training in humans: A meta-analysis. PLoS One 2013;8:e73182.

99. Tulloch H, Sweet SN, Fortier M, Capstick G, Kenny GP, Sigal RJ. Exercise facilitators and barriers from adoption to maintenance in the diabetes aerobic and resistance exercise trial [published correction appears in Can J Diabetes 2014;38:70. Sigal, Ronal J [corrected to Sigal, Ronald J]]. Can J Diabetes 2013;37:367-374. 
100. Moncion K, Biasin L, Jagroop D, et al. Barriers and facilitators to aerobic exercise implementation in stroke rehabilitation: A scoping review. J Neurol Phys Ther 2020;44:179-187.

101. Bennett H, Slattery F. Effects of blood flow restriction training on aerobic capacity and performance: A systematic review. J Strength Cond Res 2019;33:572-583.

102. Song JS, Spitz RW, Yamada Y, et al. Exercise-induced hypoalgesia and pain reduction following blood flow restriction: A brief review. Phys Ther Sport 202 1;50:89-96.

103. Liang C, Burk M, Wall A, et al. 024 Resistance exercises with blood flow restriction in patients with sporadic inclusion body myositis. J Neurol Neurosurg Psychiatry 2019;90:A9.

104. Jørgensen AN, Aagaard P, Frandsen U, Boyle E, Diederichsen LP. Blood-flow restricted resistance training in patients with sporadic inclusion body myositis: A randomized controlled trial. Scand J Rheumatol 2018;47:400-409.

105. Mattar MA, Gualano B, Perandini LA, et al. Safety and possible effects of low-intensity resistance training associated with partial blood flow restriction in polymyositis and dermatomyositis. Arthritis Res Ther 2014;16:473.

106. Douris PC, D'Agostino N, Werner WG, Petrizzo J, DiFrancisco-Donoghue J. Blood flow restriction resistance training in a recreationally active person with Parkinson's disease [published online May 13, 2020]. Physiother Theory Pract, doi: 10.1080/09593985.2020. 1762812.

107. Burkhart SS, Morgan CD, Kibler WB. The disabled throwing shoulder: Spectrum of pathology Part III: The SICK scapula, scapular dyskinesis, the kinetic chain, and rehabilitation. Arthroscopy 2003;19:641-661.

108. Burkhart SS. Internal impingement of the shoulder. Instr Course Lect 2006;55:29-34.

109. Formiga MF, Fay R, Hutchinson S, et al. Effect of aerobic exercise training with and without blood flow restriction on aerobic capacity in healthy young adults: A systematic review with meta-analysis [published correction appears in Int J Sports Phys Ther 2020;15:486]. Int J Sports Phys Ther 2020;15:175-187.

110. Incognito AV, Burr JF, Millar PJ. The effects of ischemic preconditioning on human exercise performance. Sports Med 2016;46:531-544.

111. de Groot PC, Thijssen DH, Sanchez M, et al. Ischemic preconditioning improves maximal performance in humans. Eur J Appl Physiol 2010;108:141-146.

112. Jean-St-Michel E, Manlhiot C, Li J, et al. Remote preconditioning improves maximal performance in highly trained athletes. Med Sci Sports Exerc 201 1;43:1280-1286.

113. Kido K, Suga T, Tanaka D, et al. Ischemic preconditioning accelerates muscle deoxygenation dynamics and enhances exercise endurance during the work-to-work test. Physiol Rep 2015;3:e12395.

114. Kjeld T, Rasmussen MR, Jattu T, et al. Ischemic preconditioning of one forearm enhances static and dynamic apnea. Med Sci Sports Exerc 2014;46:151-155.

115. Barbosa TC, Machado AC, Braz ID, et al. Remote ischemic preconditioning delays fatigue development during handgrip exercise. Scand J Med Sci Sports 2015;25: 356-364.

116. Franz A, Behringer M, Harmsen JF, et al. Ischemic preconditioning blunts muscle damage responses induced by eccentric exercise. Med Sci Sports Exerc 2018;50: 109-115.

117. Tanaka D, Suga $\mathrm{T}$, Tanaka $\mathrm{T}$, et al. Ischemic preconditioning enhances muscle endurance during sustained isometric exercise. Int J Sports Med 2016;37: 614-618.

118. Rubin EB, Mazzoli V, Black MS, et al. Effects of the competitive season and off-season on knee articular cartilage in collegiate basketball players using quantitative MRI: A multicenter study. J Magn Reson Imaging 2021;54:840-851.

119. Conte D, Kamarauskas P, Ferioli D, et al. Workload and well-being across games played on consecutive days during in-season phase in basketball players. J Sports Med Phys Fitness 2021;61:534-541.

120. Conte D, Kolb N, Scanlan AT, Santolamazza F. Monitoring training load and well-being during the in-season phase in National Collegiate Athletic Association Division I men's basketball. Int J Sports Physiol Perform 2018;13:1067-1074.

121. Ferioli D, Scanlan AT, Conte D, Tibiletti E, Rampinini E. The business end of the season: A comparison between playoff and regular-season workloads in professional basketball players. Int J Sports Physiol Perform 2021;16: 655-662.

122. Faltus J, Owens J, Hedt C. Theoretical applications of blood flow restriction training in managing chronic ankle instability in the basketball athlete. Int J Sports Phys Ther 2018;13:552-560.

123. Khalil LS, Okoroha KR, Jildeh TR, et al. Do anatomic changes found in the throwing arm after a season of pitching resolve with off-season rest? A dynamic ultrasound study. JSES Open Access 2019;3:338-343.

124. Chalmers PN, English J, Cushman DM, et al. The ulnar collateral ligament responds to stress in professional pitchers. J Shoulder Elbow Surg 2021;30:495-503.

125. Chan JM, Zajac J, Erickson BJ, et al. Upper extremity and hip range of motion changes throughout a season in professional baseball players. Am J Sports Med 2020;48: 481-487. 